\title{
Synthesis and Characterization of Mixed-valent
}

\section{Iron Layered Double Hydroxides (“Green Rust")}

Vincent P. Aguirre ${ }^{a}$, Simonne Jocic ${ }^{a}$, Paul Webster ${ }^{a}$, Christopher Buser ${ }^{a}$, John A. Moss ${ }^{a}$, Laura

M. Barge ${ }^{b}$, Yijie Tang ${ }^{c}$, Yisong Guo ${ }^{c}$, Marc M. Baum ${ }^{a^{*}}$

${ }^{a}$ Department of Chemistry, Oak Crest Institute of Science, 132 W. Chestnut Ave., Monrovia, CA

91016, USA

${ }^{b}$ NASA Jet Propulsion Laboratory, California Institute of Technology, 4800 Oak Grove Drive,

Pasadena CA 91109, USA

${ }^{\mathrm{c}}$ Chemistry Department, Carnegie Mellon University, 4400 Fifth Ave., Pittsburgh, PA 15213, USA

*Correspondence: m.baum@oak-crest.org, 1-626-817-0883 


\section{Supporting Information}

\section{Appendix A:}

Mössbauer spectrum (black trace) of $\mathrm{GR} 1\left(\mathrm{Cl}^{-}\right)$sample measured at $100 \mathrm{~K}$ and zero applied field (Fig. A1).

Mössbauer spectrum (black trace) of GR2 $\left(\mathrm{SO}_{4}{ }^{2-}\right)$ sample measured at $100 \mathrm{~K}$ and zero applied field (Fig. A2).

SEM images of a representative $\mathrm{GR} 1\left(\mathrm{Cl}^{-}\right)$sample air-dried under anaerobic conditions on filter paper (Fig. A3).

SEM image of an intact drop of liquid $\mathrm{GR} 1\left(\mathrm{Cl}^{-}\right)$suspension, dried in the microscope sample compartment (Fig. A4).

A droplet of a GR1 $\left(\mathrm{Cl}^{-}\right)$suspension was dried onto a carbon-coated glass coverslip in the SEM specimen chamber as it was evacuated (Fig. A5).

Inner components of the disrupted, dried droplet of $\mathrm{GR} 1\left(\mathrm{Cl}^{-}\right)$suspension (Fig. A6).

\section{Appendix B:}

Reflectance Spectroscopy Calculations and Spectral Analysis. 


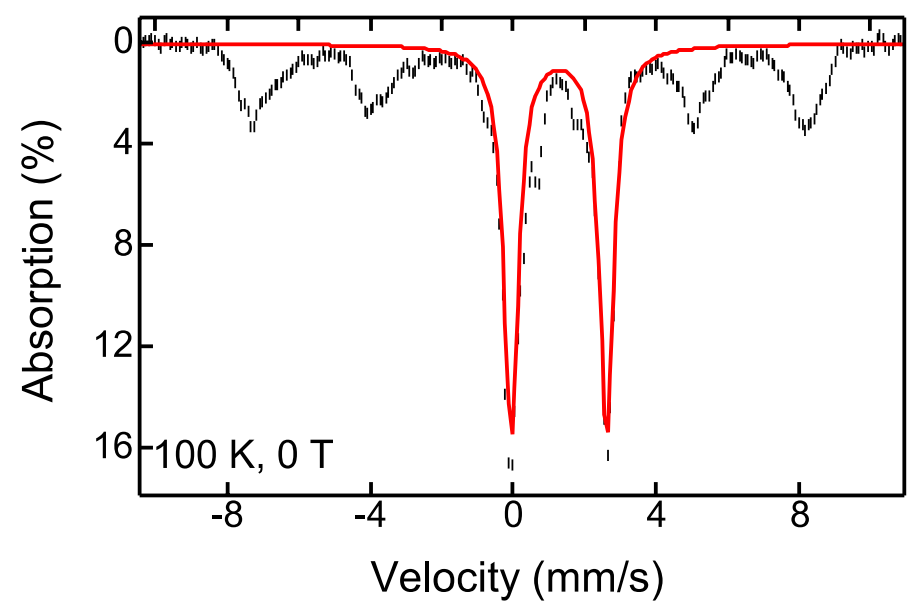

Figure A1. Mössbauer spectrum (black trace) of $\mathrm{GR} 1\left(\mathrm{Cl}^{-}\right)$sample measured at $100 \mathrm{~K}$ and zero applied field. The sample contains a quadrupole doublet with parameters $\delta=1.27 \mathrm{~mm} \mathrm{~s}^{-1}, \Delta E_{Q}=$ $2.67 \mathrm{~mm} \mathrm{~s}^{-1}, \Gamma=0.5 \mathrm{~mm} \mathrm{~s}^{-1}$ that originates from high-spin $\mathrm{Fe}(\mathrm{II})$ species and represents $50 \%$ of the total Fe in the sample. The rest of the absorption exhibits a six-line magnetic splitting pattern, typical of magnetic Fe(III) species with $\delta$ ca. $0.55 \mathrm{~mm} \mathrm{~s}^{-1}$. 


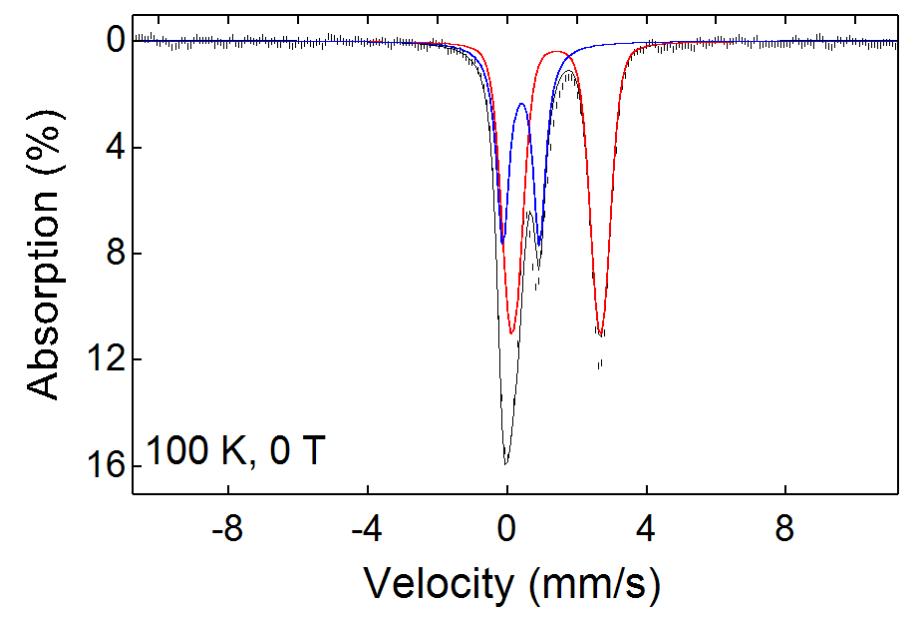

Figure A2. Mössbauer spectrum (black trace) of GR2( $\left.\mathrm{SO}_{4}{ }^{2-}\right)$ sample measured at $100 \mathrm{~K}$ and zero applied field. The spectrum can be simulated with two quadrupole doublets. The doublet I (blue trace) has the parameters of $\delta=0.39 \mathrm{~mm} \mathrm{~s}^{-1}, \Delta E q=1.03 \mathrm{~mm} \mathrm{~s}^{-1}, \Gamma=0.44 \mathrm{~mm} \mathrm{~s}^{-1}$ that represents $35 \%$ of the total Fe in the sample and is typical from Fe(III) species. The doublet II (red trace) has the parameters of $\delta=1.30 \mathrm{~mm} \mathrm{~s}^{-1}, \Delta E q=2.52 \mathrm{~mm} \mathrm{~s}^{-1}, \Gamma=0.44 \mathrm{~mm} \mathrm{~s}^{-1}$ that represents $65 \%$ of the total Fe in the sample and is typical from high-spin Fe(II) species. 


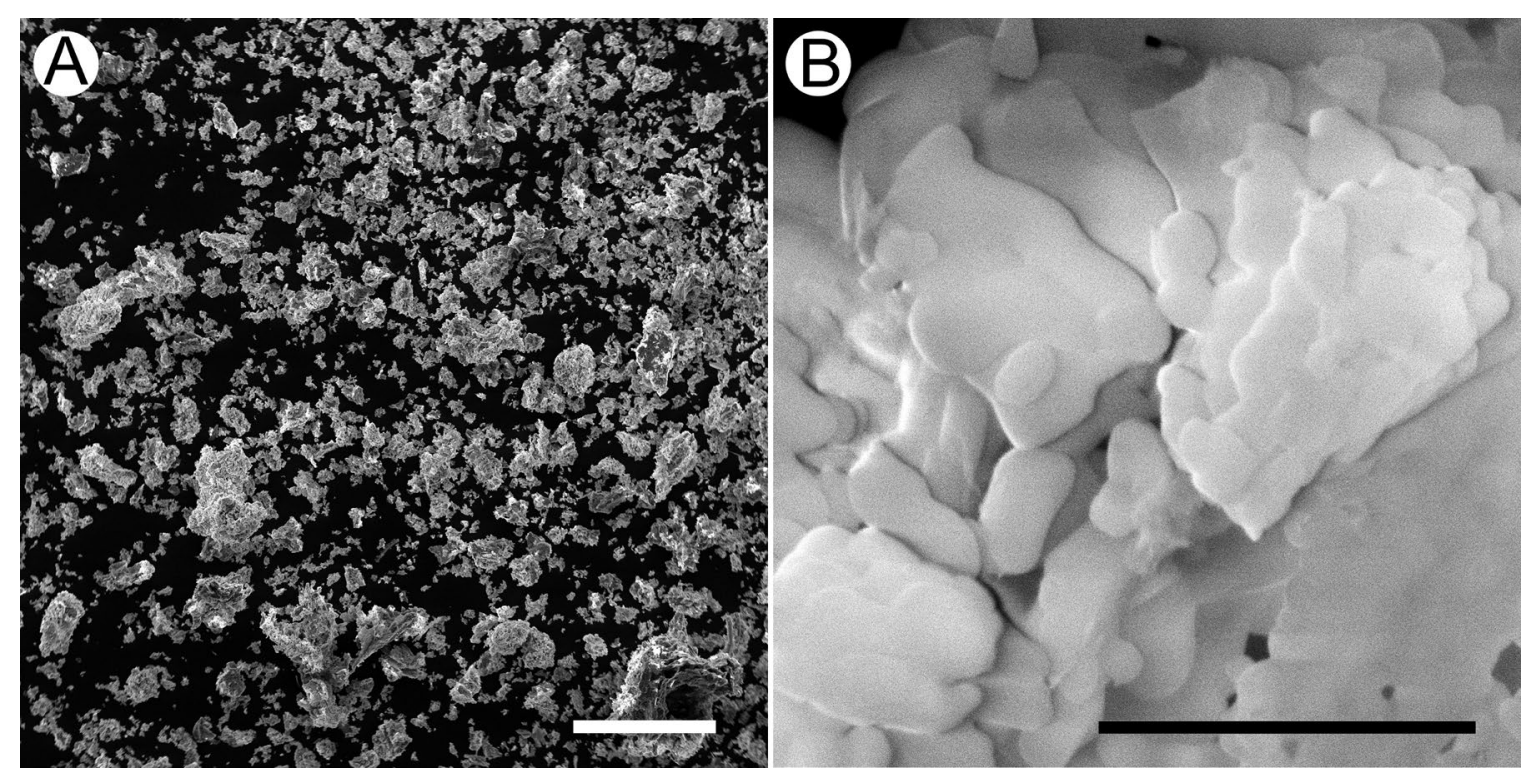

Figure A3. SEM images of a representative $\mathrm{GR} 1\left(\mathrm{Cl}^{-}\right)$sample air-dried under anaerobic conditions on filter paper. A, Low magnification image shows the particulate nature of the powder; scale bar $=500 \mu \mathrm{m}$. B, High magnification image of a particle aggregate illustrates the flat plate appearance of the components; scale bar $=5 \mu \mathrm{m}$. 


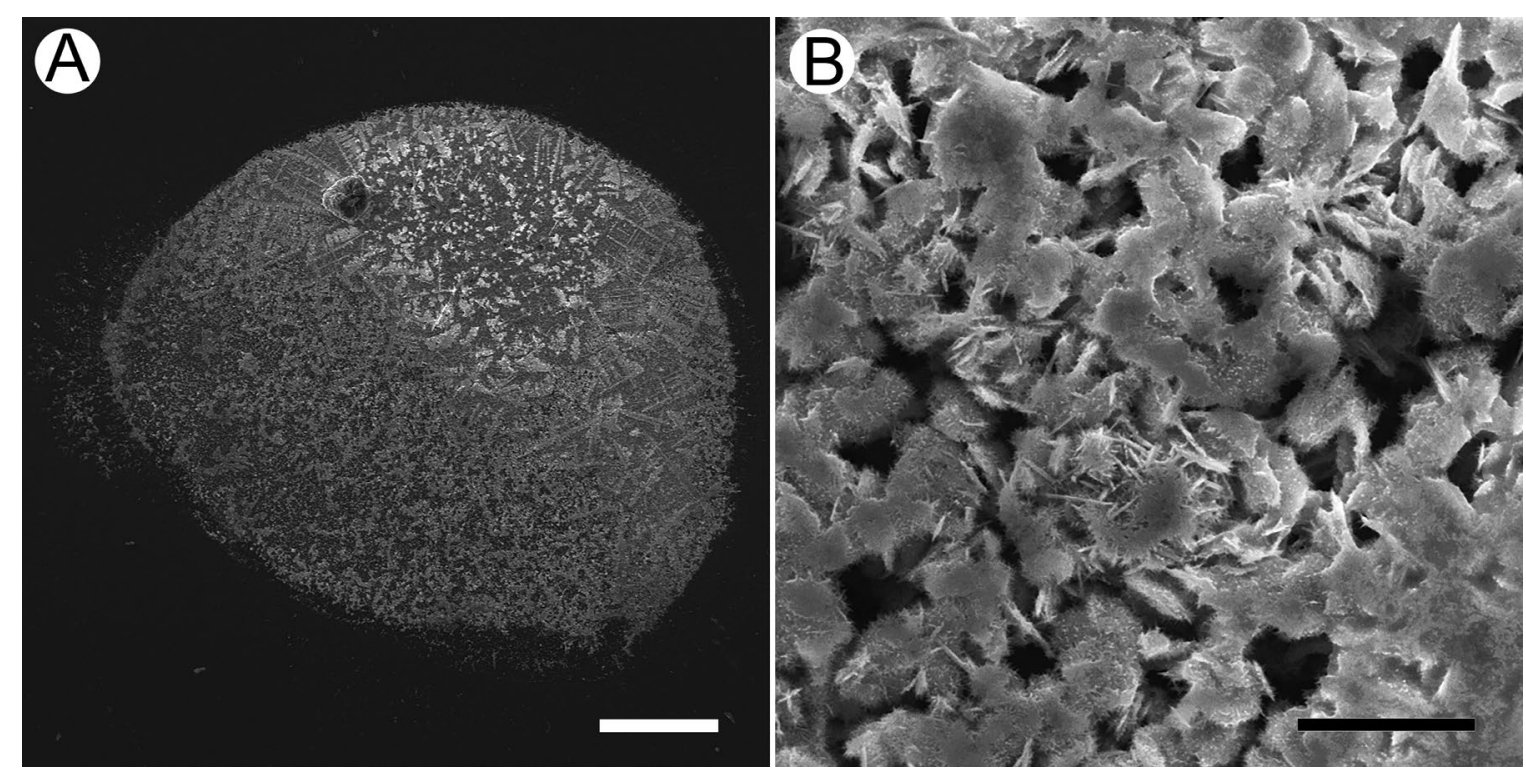

Figure A4. SEM image of an intact drop of liquid $\mathrm{GR} 1\left(\mathrm{Cl}^{-}\right)$suspension, dried in the microscope sample compartment. Oxidation of the highly air-sensitive sample upon transfer from the anaerobic transport system to the SEM vacuum chamber was observed and led to revised methods. A, Low magnification image a single drop of $\mathrm{GR} 1\left(\mathrm{Cl}^{-}\right)$suspension after drying under vacuum showing an undisturbed, crystalline outer surface; scale bar $=500 \mu \mathrm{m} . \mathbf{B}$, High magnification of the dried droplet shows surface detail, including crystals with rounded edges mixed with needle-like crystals; scale bar $=10 \mu \mathrm{m}$. 


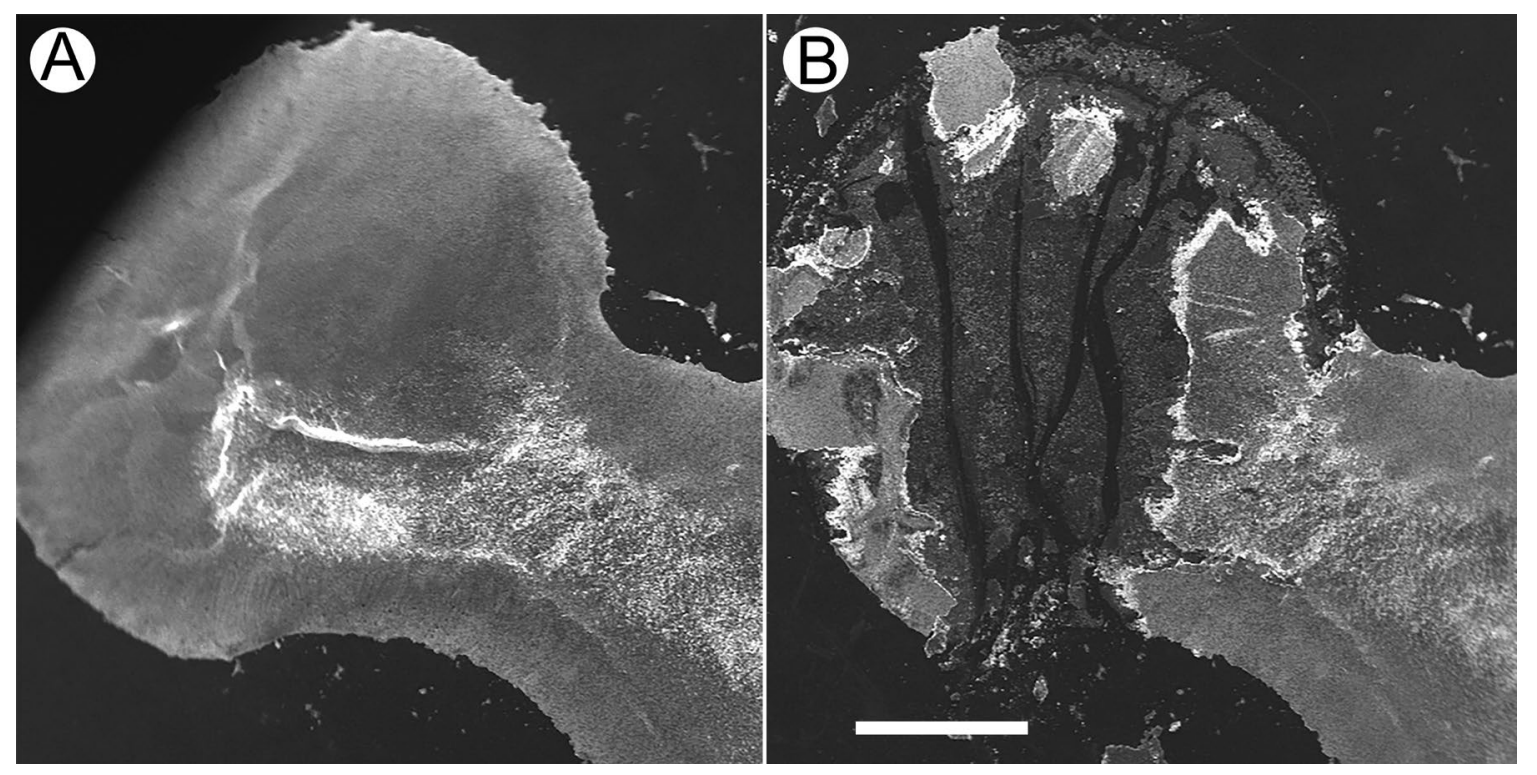

Figure A5. A droplet of a $\mathrm{GR} 1\left(\mathrm{Cl}^{-}\right)$suspension was dried onto a carbon-coated glass coverslip in the SEM specimen chamber as it was evacuated. A, Intact droplet; B. disrupted droplet; scale bar $=1 \mathrm{~mm}$. 


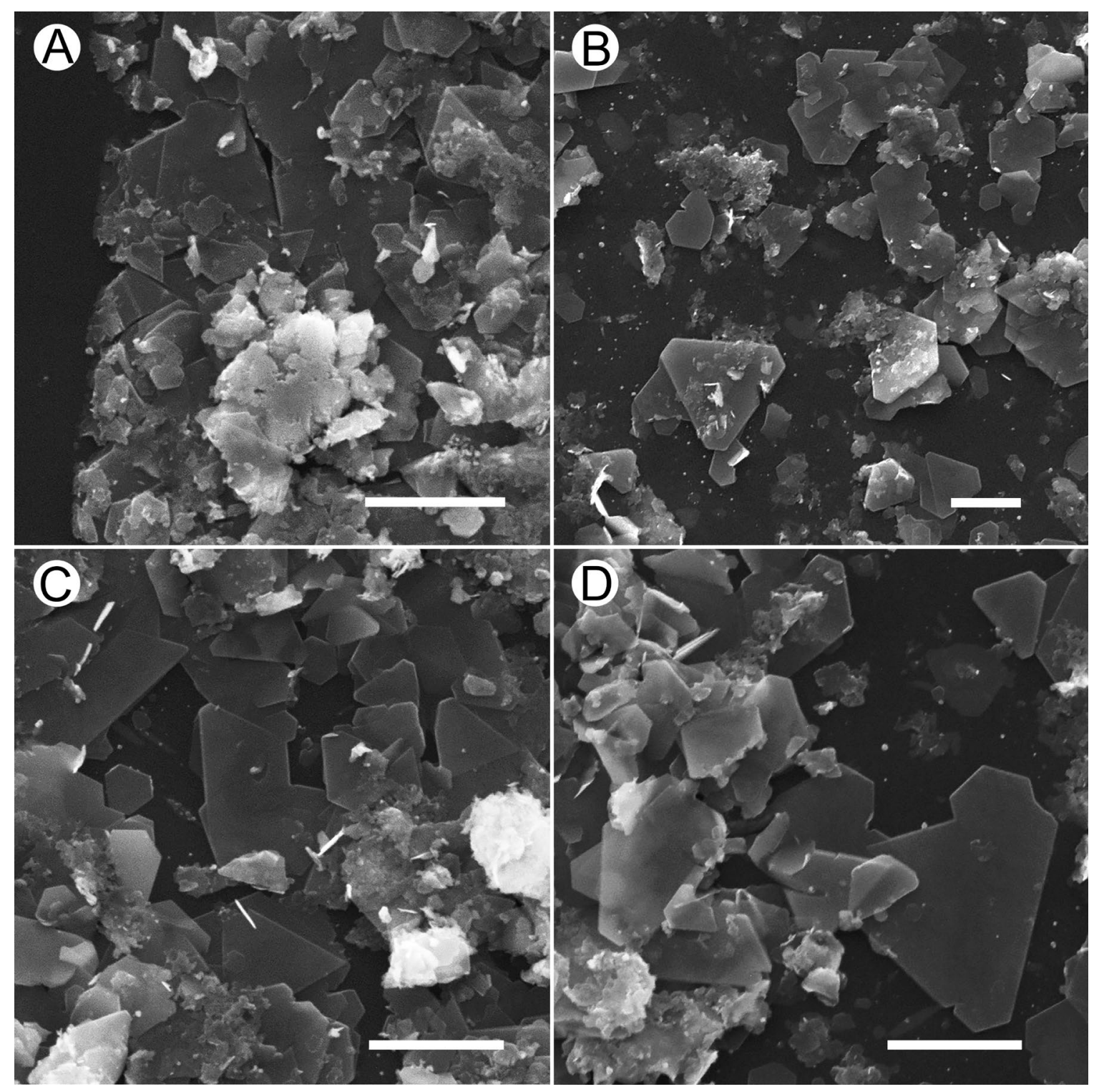

Figure A6. Inner components of the disrupted, dried droplet of $\mathrm{GR} 1\left(\mathrm{Cl}^{-}\right)$suspension shown in

Fig. A3B (center, left). A, The scraped edge where material was removed can be seen on the left of the image. Sheets of material at the base of the dried droplet were broken into irregular shapes and covered with needle-like structures and amorphous material. B, Irregular hexagonal fragments of thin, flat crystals were present on a uniformly intact, flat sheet. A few needle-like and amorphous crystals are also present. C, Image of more thin, flat irregular hexagonal-shaped crystals overlaid with needle-like structures. D, A collection of flat irregular hexagonal-shaped crystals with well-defined edges. The crystals were over a flat, unbroken sheet of material, similar in appearance to the flat crystals. All scale bars $=5 \mu \mathrm{m}$. 


\section{B. Reflectance Spectroscopy Calculations and Spectral Analysis}

Raw intensity reflectance spectra [OOIBase32 "Scope Mode" spectra in CMOS detector response (counts) versus wavelength (nm)] were imported into Microsoft Excel for data manipulation and analysis. Spectrometer response at each wavelength, $I(\lambda)$, is a function of specular and diffuse reflection of the tungsten halogen lamp output from the reaction solution/suspension and reaction flask as well as scattered room light. Scattering intensity, and consequently wavelength dependent detector response is dependent on both the solution particle density and particle size. Individual reference spectra were calculated for each reflectance spectrum using Eq. 1:

$$
I_{\text {Ref }}(\lambda)=A \cdot I_{\text {lamp }}(\lambda)+B
$$

where $I_{\text {Ref }}(\lambda)$ is the reflected intensity at each wavelength, $\lambda ; I_{\text {lamp }}(\lambda)$ is the normalized spectrum of the tungsten halogen lamp reflected off of a non-absorbing PTFE disk (in situ synthesis measurements) or a suspension of $\mathrm{MgO}$ in the measurement cuvette (decomposition reaction measurements), and $B$ is a constant that accounts for wavelength independent scattering from particles in the reaction mixture. Wavelength dependent Rayleigh scattering (elastic scattering from particles with diameter much smaller than the wavelength of light) was a negligible contributor to the reflectance spectrum and is not included in the reference spectrum calculation. The constant $A$ is a scaling factor for the lamp spectrum that is adjusted along with $B$ to generate a synthetic reference spectrum intensity value at each wavelength. For the decomposition measurements, raw intensity spectra were smoothed using a $2^{\text {nd }}$ order Savitzky-Golay filter (45 points). A fractional reflectance spectrum was computed as the ratio of each collected spectrum, $I(\lambda)$, to its synthetic reference spectrum, $I_{\text {Ref }}(\lambda)$, as shown in Eq. 2: 


$$
R_{\infty}(\lambda)=\frac{I(\lambda)}{I_{R e f}(\lambda)}
$$

Finally, the reflectance value $R_{\infty}(\lambda)$ at each wavelength was converted to Kubelka-Munk units (K-M) using Eq. 3:

$$
\mathrm{K}-\mathrm{M}=\frac{K}{S}=\frac{\left(1-R_{\infty}\right)^{2}}{2 R_{\infty}}
$$

where $K$ is the absorption coefficient and $S$ is a scattering coefficient that accounts for heterogeneous effects of light propagation and scattering in the sample. The Kubelka-Munk model describes diffuse reflectance of light from inhomogeneous materials. ${ }^{1-3}$ The value of K-M is approximately equal to the absorption coefficient and is roughly proportional to concentration. Consequently, Eq. 3 can be considered the reflectance spectroscopy analog of the absorbance transformation in transmission spectroscopy. A best fit reference spectrum for each collected reflectance spectrum was determined when areas of the spectrum showing no absorbance overlaid with the reference spectrum and gave K-M values of zero.

\section{REFERENCES}

1. Kubelka, P.; Munk, F., Ein Beitrag Zur Optik Der Farbanstriche. Z. Techn. Phys. 1931, $12,593-601$.

2. Kortüm, G., Reflectance Spectroscopy: Principles, Methods, Applications. SpringerVerlag: Berlin Heidelberg, 1969; p 366.

3. Torrent, J.; Barrón, V., Diffuse Reflectance Spectroscopy. In Methods of Soil Analysis, Part 5: Mineralogical Methods, Ulery, A. L.; Drees, L. R., Eds. Soil Science Society of America: Madison, WI, 2008; pp 367-385. 